\title{
5nm FinFET Standard Cell Library Optimization and Circuit Synthesis in Near- and Super-Threshold Voltage Regimes
}

\author{
Qing Xie, Xue Lin, Yanzhi Wang, Mohammad Javad Dousti, Alireza Shafaei, Majid Ghasemi-Gol, Massoud Pedram \\ Department of Electrical Engineering, University of Southern California, Los Angeles, CA \\ \{xqing, xuelin, yanzhiwa, dousti, shafaeib, ghasemig, pedram\}@usc.edu
}

\begin{abstract}
FinFET device has been proposed as a promising substitute for the traditional bulk CMOS-based device at the nanoscale, due to its extraordinary properties such as improved channel controllability, high $\mathrm{ON} / \mathrm{OFF}$ current ratio, reduced short-channel effects, and relative immunity to gate line-edge roughness. In addition, the near-ideal subthreshold behavior indicates the potential application of FinFET circuits in the nearthreshold supply voltage regime, which consumes an order of magnitude less energy than the regular strong-inversion circuits operating in the super-threshold supply voltage regime. This paper presents a design flow of creating standard cells by using the FinFET 5nm technology node, including both near-threshold and super-threshold operations, and building a Liberty-format standard cell library. The circuit synthesis results of various combinational and sequential circuits based on the $5 \mathrm{~nm}$ FinFET standard cell library show up to $40 \mathrm{X}$ circuit speed improvement and three orders of magnitude energy reduction compared to those of $45 \mathrm{~nm}$ bulk CMOS technology.
\end{abstract}

Keywords- FinFET; 5nm technology; standard cell library; near-threshold computing; power consumption; performance

\section{INTRODUCTION}

Energy consumption has always been a critical performance metric for integrated-circuits (ICs). The voltage down-scaling has been shown effective in reducing the energy consumption of ICs. For some relaxed-performance applications, such as portable wireless devices, medical devices, and sensor network nodes, reducing the supply voltage to a very low value, slightly higher than the threshold voltage values of transistors, results in the minimal amount of energy consumption [1][2][3]. The supply voltage that results in this minimum energy consumption, referred as the minimal energy point (MEP), has been proved and typically observed in the near-threshold voltage regime $[4][5][6]$.

The steady down-scaling of feature sizes of CMOS technology has been the driving force of the continual improvement in circuit speed and cost per functionality over the past several decades. However, due to the fundamental material and process technology limits, great challenges (i.e., how to mitigate the short-channel effects, minimize the leakage current, reduce the device-to-device variability) are

This research is supported by grants from the PERFECT program of the Defense Advanced Research Projects Agency and the Software and Hardware Foundations of the National Science Foundation. encountered during the scaling down of traditional planar CMOS transistor beyond the 22nm [7][8]. Therefore, the FinFET device, a type of quasi-planar double gate (DG) device with a process flow and layout similar to that of the traditional planar CMOS [9], has been proposed as a substitute of CMOS for future technology nodes beyond 32nm [10]. It has been reported that FinFET devices offer superior scalability [11], lower gate leakage current [12], excellent control of short-channel effects [13], and relative immunization to the gate line-edge roughness [14].

Due to the promising future of FinFET device at the nanoscale, considerable research efforts have been invested in modeling and characterizing FinFET devices. Sinha et. al. presented a predictive technology model for multi-gate transistors (PTM-MG) for FinFETs in sub-20nm technology nodes [15]. This model is based on BSIM-CMG model [16]. An alternative approach based on the fundamental physics principle is adopted by Gupta et. al, and generates FinFET device models at $5 \mathrm{~nm}$ [17]. The device model in [17] is specified by using look-up-tables (LUTs) and is compatible with SPICE through a Verilog-A interface. To predict the trend of circuit behaviors of future deeply scaled FinFET devices, we consider the most advanced FinFET technology node and adopt the $5 \mathrm{~nm}$ FinFET device model developed in [17]. In this work, we build a Synopsys Liberty-format standard cell library [18], which is widely used for logic synthesis and static timing analysis, using the 5nm FinFET technology node.

The contribution of this work is in three-fold. First, we create standard cells, including both combinational logic cells and sequential logic cells, by using $5 \mathrm{~nm}$ FinFET devices. The sizing of a standard cell is derived by properly setting the number of N-type fins and P-type fins that produce almost equal rise and fall times. Second, we characterize the $5 \mathrm{~nm}$ FinFET combinational and sequential standard cells at two supply voltages in near- and super-threshold regimes, respectively, and build the multi- $V_{d d}$ standard cell library by using the Liberty format. The timing, power, and capacitance parameters of the standard cells are measured by using HSPICE and stored in 2D LUTs. Third, we predict the timing and power performance of $5 \mathrm{~nm}$ FinFET technology by synthesizing various combinational and sequential benchmark circuits using the Synopsys Design Compiler and the characterized 5nm FinFET standard cell library. Compared to 
baseline circuits synthesized using 45nm CMOS technology, the $5 \mathrm{~nm}$ FinFET technology improves the circuit speed by up to $40 \mathrm{X}$ and reduces the energy consumption by three orders of magnitude.

The rest of this paper is organized as follows. Section II introduces the properties of $5 \mathrm{~nm}$ FinFET devices at multiple supply voltages. Section III explains the standard cell sizing. The library format and characterization flow are elaborated in Section IV. We show the synthesis results in Section V and conclude the paper in Section VI.

\section{5NM FINFET TECHNOLOGY NODE}

Figure 1 shows the structure of a $5 \mathrm{~nm}$ FinFET device. The FinFET device consists of a thin silicon body, with thickness of $T_{\text {fin }}$, which is wrapped by gate electrodes. The device is termed quasi-planar as the current flows parallel to the wafer plane, and the channel is formed perpendicular to the plane. The effective gate length $L_{G}$ is twice as large as the fin height $h_{\text {fin }}$. The spacer length $L_{S P}$ is an important design parameter that directly relates to the short channel effects [17]. Each fin has two gates: a front gate and a back gate. The FinFET device allows independent control of the front and back gates by etching away the gate electrode at the top of the channel. The FinFET device model developed in [17] considers a shorted-gate mode, in which the front gate and back gate are tied together to achieve the highest drive strength [8]. Note that in this work we focus on the shorted-gate FinFET devices as the independent gate devices suffer from many fabrication issues in practice.

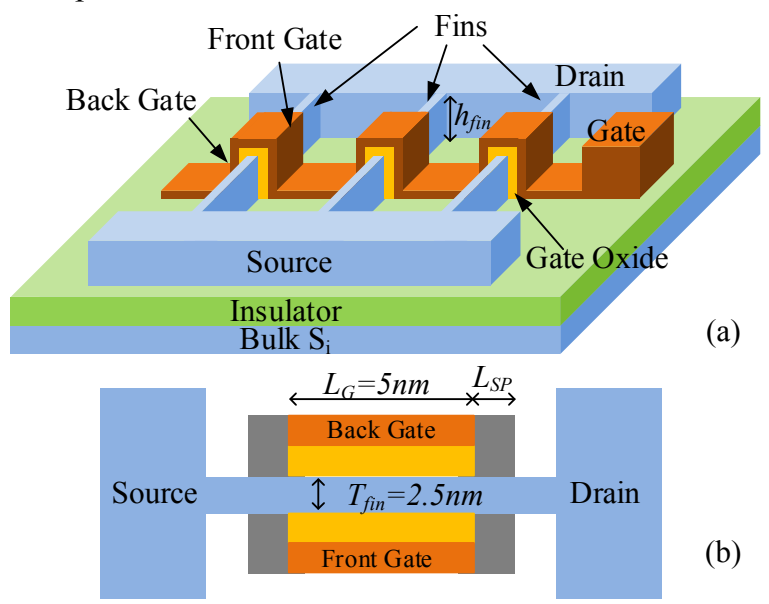

Figure 1. (a) Perspective view and (b) top view [17] of the $5 \mathrm{~nm}$ FinFET device.

Operating circuits in the near-threshold supply voltage regime results in a reduced energy consumption at the cost of circuit speed degradation. When the supply voltage $V_{d d}$ is reduced, the dynamic energy consumption reduces quadratically. However, the leakage energy consumption, which is the product of leakage power and circuit delay, increases, because the increase of the circuit delay (satisfying an exponential relation versus $V_{d d}$ ) surpasses the reduction of leakage power (satisfying a linear relation versus $V_{d d}$ ). Figure 2 shows the energy consumption of a 20 -stage inverter chain built with the $5 \mathrm{~nm}$ FinFET devices at different supply voltages. One can observe from Figure 2 that the minimal energy point for typical circuit operations occurs near $0.2 \mathrm{~V}$, which is less than the threshold value of the FinFET device $(0.2 \mathrm{~V} \sim 0.25 \mathrm{~V})$. To enable both low power and high performance applications, we build a standard cell library that includes two supply voltages: $0.3 \mathrm{~V}$ for near-threshold regime and $0.45 \mathrm{~V}$ for super-threshold regime.

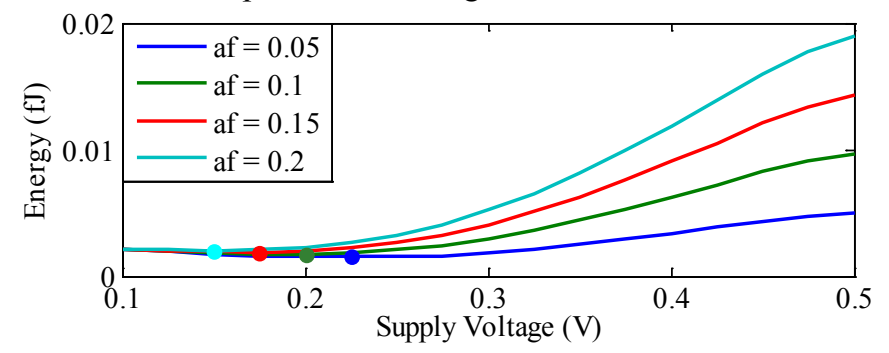

Figure 2. MEPs (denoted by dots) of a 20-stage 5nm FinFET inverter chain at different activity factors.

\section{CReating Standard Cells}

As shown in Figure 1 (a), the drive strength of a FinFET device depends on the ratio of fin height and channel length, whereas both parameters are determined by the fabrication technology. Thus, the FinFET standard cell sizing is to select the appropriate number of fins for the pull-up and pull-down network of each logic cell.

\section{A. Inverter Sizing}

First we investigate the numbers of P-type fins and N-type fins in an inverter that achieves approximately equal rise and fall delays. According to the trans-regional FinFET model [6], the drain current of a FinFET in the sub- and near-threshold regions is given by

$$
I_{d s}=I_{0} e^{\frac{\left(V_{g s}+\lambda V_{d s}-V_{t h}\right)-a\left(V_{g s}+\lambda V_{d s}-V_{t h}\right)^{2}}{m \cdot v_{T}}}\left(1-e^{\frac{-V_{d s}}{v_{T}}}\right)
$$

where $\lambda$ is the drain voltage dependence coefficient (similar to, but much smaller than, the DIBL coefficient for bulk CMOS devices), $v_{T}$ is the thermal voltage, and $I_{0}, a$, and $m$ are technology-dependent parameters to be extracted from HSPICE simulation.

In order to achieve equal rise and fall delay, the number of P-type fins $N_{P}$ in an inverter can be determined by

$$
N_{P}=N_{N} \cdot \frac{I_{d s, N}}{I_{d s, P}}
$$

where $I_{d s, N}$ is the drain current of an N-type fin when $V_{g s}=V_{d s}=V_{D D}, I_{d s, P}$ is the drain current of a P-type fin when $\left|V_{g s}\right|=\left|V_{d s}\right|=V_{D D}$, and $N_{N}$ is the number of N-type fins in the inverter.

Based on (2) and HSPICE simulation, the $N_{P} / N_{N}$ ratios of INV1X, INV2X, INV4X, and INV8X gates in the nearthreshold region $\left(V_{D D}=0.3 \mathrm{~V}\right)$ are $1 / 1,2 / 2,4 / 4$, and 8/7, respectively. Note that we round to the nearest integer number of fins to achieve almost equal driving strengths of the pull-up and pull-down network. 


\section{B. Stack Sizing of FinFET Logic Cells}

In order to design other combinational logic cells under near-threshold region, we need to solve the stack sizing problem. In some logic cells, there are several transistors connected in series forming a stack, e.g., the pull-down network of a NAND or the pull-up network of a NOR. The stack sizing problem involves determining the transistor sizes in a stack such that the logic cell achieves equal rise and fall delays. We use the 2-input NAND1X as an example. Figure 3 shows an INV1X and a 2-input NAND1X, and the number on top of a FinFET transistor symbol denotes the number of parallel- connected fins in that FinFET transistor. The INV1X achieves equal rise and fall delays in the near-threshold region. We denote the stack sizing factor in an $m$-input NAND by $\rho_{N, m}$, where the subscript $N$ denotes an N-type FinFET device. Similarly, the stack sizing factor in an $m$-input NOR is denoted by $\rho_{P, m}$. The stack sizing factor $\rho_{N, 2}$ of the 2input NAND is defined as the ratio of the number of N-type fins connected to an input signal in the 2-input NAND1X to this number in the INV1X, such that the pull down network of the 2-input NAND1X has the same current driving strength as it has in the INV1X. From a theoretical calculation based on FinFET model in (1) and HSPICE simulation, we obtain $\rho_{N, 2}=3.25 \approx 3$ in the near-threshold region. Please note that $\rho_{N, 2}$ is larger than 2 , which is the typical value for bulk CMOS in the super-threshold region. Similarly, we obtain $\rho_{P, 2}=3, \rho_{N, 3}=6$, and $\rho_{P, 3}=5$. Please note that a stack of more than three transistors may not be favored in the nearthreshold circuits because of the significant performance degradation.
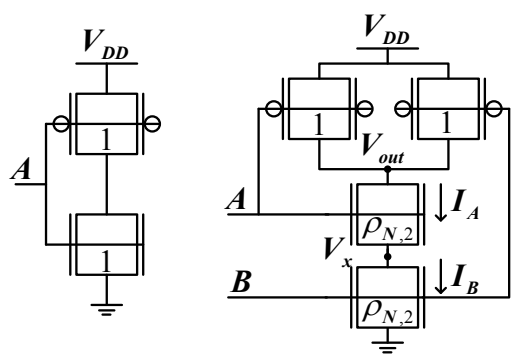

Figure 3. Illustration of stack sizing for a 2-input NAND.

\section{Combinational and Sequential Logic Cell Sizing}

Similar to the sizing of INV's and derived stack sizing factors, we derive the sizing of all other combinational logic cells and the sequential logic cells accordingly. All the logic cells included in the $5 \mathrm{~nm}$ FinFET standard cell library are summarized in Table 1 . The functionality of each logic cell is verified by HSPICE simulation. Please note that we use the same sizing of FinFET logic cells in the super-threshold region $\left(V_{D D}=0.45 \mathrm{~V}\right)$, since we assume our standard cells support DVFS (dynamic voltage and frequency scaling).

Table 1. Logic cells in 5nm FinFET standard cell library.

\begin{tabular}{|c|c|c|}
\hline & Cell type & Scale/triggering \\
\hline \multirow{2}{*}{$\begin{array}{c}\text { Combinational } \\
\text { logic cells }\end{array}$} & Inverter & $1 \mathrm{X}, 2 \mathrm{X}, 4 \mathrm{X}, 8 \mathrm{X}$ \\
\cline { 2 - 3 } & 2-input NAND & $1 \mathrm{X}, 2 \mathrm{X}, 4 \mathrm{X}, 8 \mathrm{X}$ \\
\cline { 2 - 3 } & 3-input NAND & $1 \mathrm{X}, 2 \mathrm{X}, 4 \mathrm{X}$ \\
\hline
\end{tabular}

\begin{tabular}{|c|c|c|}
\hline \multirow{7}{*}{} & 2-input NOR & $1 \mathrm{X}, 2 \mathrm{X}, 4 \mathrm{X}, 8 \mathrm{X}$ \\
\cline { 2 - 3 } & 3-input NOR & $1 \mathrm{X}, 2 \mathrm{X}, 4 \mathrm{X}$ \\
\cline { 2 - 3 } & AND-OR-INV & $1 \mathrm{X}, 2 \mathrm{X}, 4 \mathrm{X}$ \\
\cline { 2 - 3 } & OR-AND-INV & $1 \mathrm{X}, 2 \mathrm{X}, 4 \mathrm{X}$ \\
\cline { 2 - 3 } & XNOR & $1 \mathrm{X}, 2 \mathrm{X}$, \\
\cline { 2 - 3 } & XOR & $1 \mathrm{X}, 2 \mathrm{X}$, \\
\cline { 2 - 3 } $\begin{array}{c}\text { Sequential } \\
\text { logic cells }\end{array}$ & MUX & Active-high \\
\cline { 2 - 3 } & Latch & Positive-edge \\
\cline { 2 - 3 } & D-flip-flop w/ S/R & $\begin{array}{c}\text { Positive-edge with } \\
\text { asynchronous set } \\
\text { and reset signals }\end{array}$ \\
\hline
\end{tabular}

\section{STANDARD CELL LIBRARY CHARACTERIZATION}

\section{A. Liberty-format Standard Cell Library}

A standard cell library is a set of high quality timing and power models that accurately and efficiently capture behaviors of standard cells. The standard cell library is widely used in many design tools for different purposes, such as logic synthesis, static timing analysis, power analysis, high-level design language simulation, and so on, in the computer-aideddesign (CAD) domain. The Liberty library format (.lib), which was first invented by Synopsys one decade ago, has become an industrial standard that is adopted by over 100 semiconductor vendors and implemented in over 75 production electronic design automation (EDA) tools [18]. Therefore, we build our $5 \mathrm{~nm}$ FinFET standard cell library in the lib format.

The Liberty library is built in a hierarchical manner, as shown in Figure 4. The information of process, supply voltage, data units, LUT template, triggering thresholds, and so on, is specified in the library-level. The library contains a number of standard cells, including both combinational and sequential cells with different scales. In the cell-level, the cell area, leakage power, and each individual input/output pin are specified. The signal direction, symbolic function, and input capacitance are listed for each pin. In addition, the timing parameters (propagation delay, output slew, and timing check values) and power parameters (internal power) are also stored in 2D LUTs in the pin-level. We obtain those timing and power parameters of each logic cell of interest through HSPICE simulations at various input and output conditions based on the Verilog-A based 5nm FinFET device model.

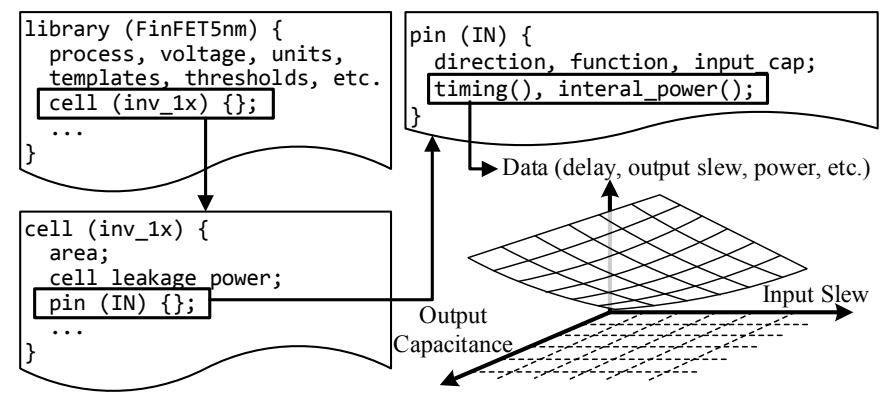

Figure 4. Hierarchy of Liberty format library. 
Table 2. Circuit delay and energy consumption of combinational benchmarks.

\begin{tabular}{|c|c|c|c|c|c|c|c|c|c|c|c|c|}
\hline & \multicolumn{5}{|c|}{ Circuit Delay (ns) } & \multicolumn{5}{c|}{ Energy consumption (fJ) } \\
\hline Library & $\begin{array}{c}\text { FinFET } \\
5 \mathrm{~nm}\end{array}$ & $\begin{array}{c}\text { FinFET } \\
5 \mathrm{~nm}\end{array}$ & $\begin{array}{c}\text { CMOS } \\
16 \mathrm{~nm}\end{array}$ & $\begin{array}{c}\text { CMOS } \\
16 \mathrm{~nm}\end{array}$ & $\begin{array}{c}\text { Nangate } \\
45 \mathrm{~nm}\end{array}$ & $\begin{array}{c}\text { NCSU } \\
45 \mathrm{~nm}\end{array}$ & $\begin{array}{c}\text { FinFET } \\
5 \mathrm{~nm}\end{array}$ & $\begin{array}{c}\text { FinFET } \\
5 \mathrm{~nm}\end{array}$ & $\begin{array}{c}\text { CMOS } \\
16 \mathrm{~nm}\end{array}$ & $\begin{array}{c}\text { CMOS } \\
16 \mathrm{~nm}\end{array}$ & $\begin{array}{c}\text { Nangate } \\
45 \mathrm{~nm}\end{array}$ & $\begin{array}{c}\text { NCSU } \\
45 \mathrm{~nm}\end{array}$ \\
\hline$V_{d d}(\mathrm{~V})$ & 0.30 & 0.45 & 0.50 & 0.70 & 1.10 & 1.10 & 0.30 & 0.45 & 0.50 & 0.70 & 1.10 & 1.10 \\
\hline $\mathrm{c} 499$ & 0.071 & 0.024 & 0.501 & 0.213 & 0.6 & 0.6 & 0.16 & 0.95 & 27.95 & 65.37 & 590.4 & 997.3 \\
\hline $\mathrm{c} 1355$ & 0.099 & 0.034 & 0.548 & 0.210 & 1.02 & 1.04 & 0.32 & 1.44 & 25.43 & 62.67 & 1,235 & 671.4 \\
\hline c1908 & 0.107 & 0.039 & 0.757 & 0.328 & 1.26 & 1.22 & 0.16 & 0.76 & 23.00 & 57.31 & 905.77 & 507.6 \\
\hline c3540 & 0.146 & 0.048 & 0.857 & 0.390 & 1.79 & 1.48 & 0.65 & 2.42 & 58.07 & 121.4 & 2,395 & 1,182 \\
\hline 16-bit adder & 0.153 & 0.051 & 1.109 & 0.404 & 1.45 & 1.31 & 0.053 & 0.33 & 4.51 & 10.12 & 102.5 & 219.8 \\
\hline 16-bit multiplier & 0.242 & 0.071 & 2.827 & 0.970 & 2.89 & 2.71 & 1.64 & 7.41 & 262.8 & 598.1 & 3,300 & 6,300 \\
\hline
\end{tabular}

\section{B. Library Characterization}

1) Characterizing timing parameters: The timing parameters of a logic cell refer to propagation delays and transition times of the output pin when the output makes a transition. For sequential cells such as D flip-flops and latches, the timing parameters also include time check parameters such as the setup time and hold time of the data signal, and the recovery time and removal time of asynchronous control signals. The propagation delays and transition times are represented by using 2D LUTs, which are indexed by input transition times and output load capacitance at the output pin, as shown in Figure 4. The design tools evaluate the path delay in a circuit by indexing the delay LUTs using the total fanout load capacitance and transition time of the input pin, which are obtained by indexing transition time LUTs in the previous stage. The time check parameters of sequential cells are independent of the load capacitances, and thus they are indexed by transition times of data or control signal and transition times of the clock signal instead.

We apply the single input switching (SIS) assumption such that only one input signal switches at a time. Therefore, propagation delays and transition times are measured for output pin related to each input pin, while signals of other input pins stay unchanged. We define the propagation delay as the time interval from the moment that the triggering signal of the related input pin crosses the $50 \%$ of $V_{d d}$ to the moment that the output signal crosses the $50 \%$ of $V_{d d}$. The output transition time is measured as the time that the output voltage takes to transit from $20 \%$ to $80 \%$ of $V_{d d}$ for rising and from $80 \%$ to $20 \%$ of $V_{d d}$ for falling. For flip-flops, the related input pin is the clock pin, while for latches, both clock pin and data pin can be the related input pin.

Setup time and hold time are important timing constraints for sequential cells that shall be satisfied to ensure correct circuit functionality. To measure the setup time and hold time, we start from an initial time range and apply the bisection method [19] that binarily reduces the time range to locate the target value, above which the timing failure of the sequential logic cell occurs. We record setup times and hold times in 2D LUTs. Similarly, recovery time and removal time are defined for asynchronous control signal, which can be analogous to setup time and hold time of the data signal [20]. We measure recovery times and removal times by using the bisection method and store them in 2D LUTs indexed by transition times of clock signal and asynchronous control signal.

2) Characterizing the power parameters: The power parameters in the Liberty library include the leakage power and internal power of a logic cell. The overall power consumption is evaluated by summing up the leakage power, internal power, and switching power (power consumed when charging and discharging the load capacitance.) We measure the leakage power consumption by multiplying the supply voltage to the average current flowing out from the $V_{d d}$ terminal when there is no input and output signal transition. The internal power accounts for the short-circuit power consumption and dynamic power of the diffusion capacitors at the output pin of the logic cell. For combinational logic cells, the internal power is measured by subtracting the switching energy at the load capacitance from the total energy consumption when output signal transits. For sequential logics, we measure the swtiching power of output pin, input data pin, and clock pin. 2D LUTs, similar to the one shown in Figure 4, are used to store internal power values of the output pin related to each input pin.

3) Characterizing the input capacitance: In liberty library, the total fanout load capacitance of a logic cell is calculated by summing up the input capacitance of its fanout cells. For every input pin of each logic cell, we characterize the input capacitance by dividing the integral of the driving current of the input pin over the time that input signal switches by the supply voltage.

\section{SYNTHESIS RESUlTS}

We synthesize various combinational and sequential benchmark circuits, as well as the LEON2 processor [21] by using the developed 5nm FinFET standard cell library. To show the circuit speed improvement and energy reduction at this technology node, we compare the timing and energy results of same circuits synthesized by using different CMOS standard cell library at $16 \mathrm{~nm}$ and $45 \mathrm{~nm}$. We build the $16 \mathrm{~nm}$ multi- $V_{d d}$ CMOS standard cell library $(0.7 \mathrm{~V}$ for high performance usage and $0.5 \mathrm{~V}$ for low power usage) by 
Table 3. Energy-delay product of combinational benchmarks.

\begin{tabular}{|c|c|c|c|c|c|c|}
\hline & \multicolumn{6}{|c|}{ Energy-delay product (fJ-ns) } \\
\hline Library & $\begin{array}{c}\text { FinFET } \\
5 \mathrm{~nm}\end{array}$ & $\begin{array}{c}\text { FinFET } \\
5 \mathrm{~nm}\end{array}$ & $\begin{array}{c}\text { CMOS } \\
16 \mathrm{~nm}\end{array}$ & $\begin{array}{c}\text { CMOS } \\
16 \mathrm{~nm}\end{array}$ & $\begin{array}{c}\text { Nangate } \\
45 \mathrm{~nm}\end{array}$ & $\begin{array}{c}\text { NCSU } \\
45 \mathrm{~nm}\end{array}$ \\
\hline$V_{d d}(\mathrm{~V})$ & 0.30 & 0.45 & 0.50 & 0.70 & 1.10 & 1.10 \\
\hline $\mathrm{c} 499$ & 0.011 & 0.02. & 14.00 & 13.92 & 354.2 & 598.4 \\
\hline $\mathrm{c} 1355$ & 0.032 & 0.049 & 13.94 & 13.16 & 1,259 & 698.3 \\
\hline $\mathrm{c} 1908$ & 0.017 & 0.030 & 17.41 & 18.80 & 1,141 & 619.3 \\
\hline $\mathrm{c} 3540$ & 0.095 & 0.116 & 49.77 & 47.35 & 4,287 & 1,749 \\
\hline 16-bit adder & 0.008 & 0.017 & 5.002 & 4.088 & 148.6 & 287.9 \\
\hline 16-bit multiplier & 0.397 & 0.526 & 742.9 & 580.2 & 9,537 & 17,073 \\
\hline
\end{tabular}

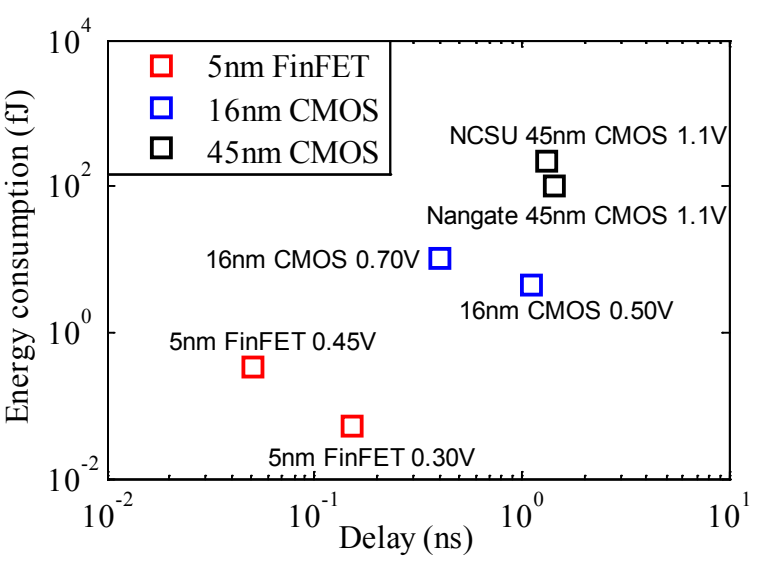

Figure 5. Energy vs. circuit delay for 16-bit adder at different technology nodes.

Table 4. Frequency and power consumption of sequential benchmarks.

\begin{tabular}{|c|c|c|c|c|c|c|c|c|c|c|c|c|}
\hline & \multicolumn{5}{|c|}{ Frequency $(\mathrm{GHz})$} & \multicolumn{5}{c|}{ Power consumption (uW) } \\
\hline Library & $\begin{array}{c}\text { FinFET } \\
5 \mathrm{~nm}\end{array}$ & $\begin{array}{c}\text { FinFET } \\
5 \mathrm{~nm}\end{array}$ & $\begin{array}{c}\text { CMOS } \\
16 \mathrm{~nm}\end{array}$ & $\begin{array}{c}\text { CMOS } \\
16 \mathrm{~nm}\end{array}$ & $\begin{array}{c}\text { Nangate } \\
45 \mathrm{~nm}\end{array}$ & $\begin{array}{c}\text { NCSU } \\
45 \mathrm{~nm}\end{array}$ & $\begin{array}{c}\text { FinFET } \\
5 \mathrm{~nm}\end{array}$ & $\begin{array}{c}\text { FinFET } \\
5 \mathrm{~nm}\end{array}$ & $\begin{array}{c}\text { CMOS } \\
16 \mathrm{~nm}\end{array}$ & $\begin{array}{c}\text { CMOS } \\
16 \mathrm{~nm}\end{array}$ & $\begin{array}{c}\text { Nangate } \\
45 \mathrm{~nm}\end{array}$ & $\begin{array}{c}\text { NCSU } \\
45 \mathrm{~nm}\end{array}$ \\
\hline$V_{d d}(\mathrm{~V})$ & 0.30 & 0.45 & 0.50 & 0.70 & 1.10 & 1.10 & 0.30 & 0.45 & 0.50 & 0.70 & 1.10 & 1.10 \\
\hline $\mathrm{s} 820$ & 25 & 50 & 7.7 & 14.3 & 2.5 & 2.5 & 4.5 & 31.1 & 112.8 & 477.9 & 735.1 & 1,483 \\
\hline $\mathrm{s} 1423$ & 10 & 20 & 3.3 & 6.7 & 1.1 & 1.0 & 4.2 & 30.7 & 135.3 & 568.9 & 984.6 & 1,381 \\
\hline Arbiter & 10 & 25 & 2.9 & 5.0 & 1.1 & 1.0 & 4.3 & 26.9 & 42.7 & 225.2 & 888.3 & 2,235 \\
\hline LEON2 SPARC & 2.5 & 6.7 & 1.0 & 2.5 & 0.48 & 0.48 & 51 & 268.2 & 764.2 & 4,785 & 8,173 & 16,658 \\
\hline
\end{tabular}

Table 5. Energy per clock cycle of sequential benchmarks.

\begin{tabular}{|c|c|c|c|c|c|c|}
\hline & \multicolumn{5}{|c|}{ Energy per clock cycle (fJ) } \\
\hline Library & $\begin{array}{c}\text { FinFET } \\
5 \mathrm{~nm}\end{array}$ & $\begin{array}{c}\text { FinFET } \\
5 \mathrm{~nm}\end{array}$ & $\begin{array}{c}\text { CMOS } \\
16 \mathrm{~nm}\end{array}$ & $\begin{array}{c}\text { CMOS } \\
16 \mathrm{~nm}\end{array}$ & $\begin{array}{c}\text { Nangate } \\
45 \mathrm{~nm}\end{array}$ & $\begin{array}{c}\text { NCSU } \\
45 \mathrm{~nm}\end{array}$ \\
\hline$V_{d d}(\mathrm{~V})$ & 0.30 & 0.45 & 0.50 & 0.70 & 1.10 & 1.10 \\
\hline $\mathrm{s} 820$ & 0.18 & 0.62 & 14.65 & 33.42 & 294.0 & 593.2 \\
\hline $\mathrm{s} 1423$ & 0.42 & 1.54 & 41.00 & 84.91 & 895.1 & 1,381 \\
\hline Arbiter & 0.43 & 1.08 & 14.72 & 45.04 & 807.5 & 2,235 \\
\hline LEON2 SPARC & 20.4 & 40.03 & 764.2 & 1,914 & 17,027 & 34,704 \\
\hline
\end{tabular}

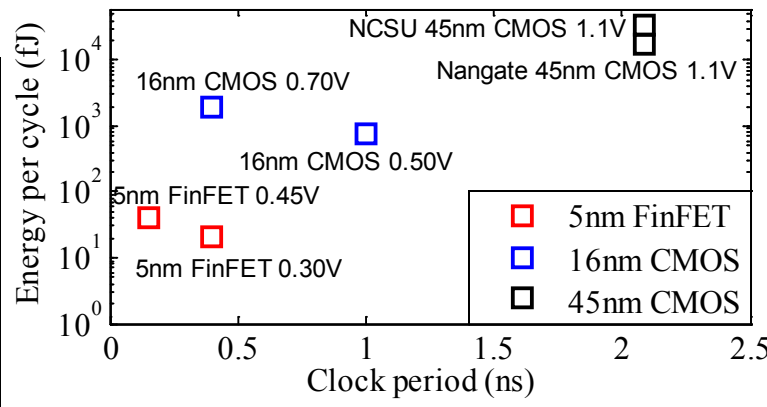

Figure 6. Energy per clock cycle vs. clock period for LEON2 SPARC at different technology nodes. following the same design flow elaborated in Section III and IV, based on the $16 \mathrm{~nm}$ PTM CMOS device model [15]. The $45 \mathrm{~nm}$ standard cell libraries include a commercial library developed by Nangate Inc. [23] and a library freely distributed by North Carolina State University [24]. All benchmark circuits are synthesized by using Synopsys Design Compiler [25].

Table 2 compares the delay of some combinational benchmarks, containing several ISCAS combinational benchmark circuits, a 16-bit carry-ripple-adder, and a 16-bit binary multiplier. From Table 2, one may observe that at the nominal supply voltage of each technology node, operating $5 \mathrm{~nm}$ FinFET circuits at $0.45 \mathrm{~V}$ achieves circuit speed improvement by a factor of $8 \mathrm{X} \sim 14 \mathrm{X}$ against $16 \mathrm{~nm}$ CMOS circuits at $0.7 \mathrm{~V}$, and $25 \mathrm{X} \sim 41 \mathrm{X}$ against $45 \mathrm{~nm}$ CMOS circuits at $1.1 \mathrm{~V}$, respectively. In low power mode, operating $5 \mathrm{~nm}$ FinFET circuits at $0.3 \mathrm{~V}$ results in $7 \mathrm{X} \sim 12 \mathrm{X}$ circuit speed improvement against that of $16 \mathrm{~nm}$ CMOS circuits at $0.5 \mathrm{~V}$.

The corresponding energy consumptions at each technology node for those combinational benchmarks are also summarized in Table 2. One can observe that in the nominal condition, 5nm FinFET circuits results in 30X $\sim 80 \mathrm{X}$ energy reduction against $16 \mathrm{~nm}$ CMOS circuits, and $666 \mathrm{X} \sim 1050 \mathrm{X}$ energy reduction against $45 \mathrm{~nm}$ CMOS circuits, respectively. In addition, operating 5nm FinFET circuits in low-power mode results in $85 \mathrm{X} \sim 174 \mathrm{X}$ energy reduction against $16 \mathrm{~nm}$ CMOS circuits.

An important property of FinFET devices comes from the high $I_{o n} / I_{\text {off }}$ ratio, which results in higher ratio between dynamic energy consumption and leakage energy 
consumption, comparing with that of CMOS circuits. It is more energy efficient to operate FinFET circuits at low $V_{d d}$ as we can save dynamic energy, which is the dominant part for FinFET circuits, in the near-threshold regime. More energy reduction is observed in Table 2 for FinFET circuits when we compare energy results obtained in nominal condition and low-power condition. For example, we observe 6X energy reduction with $3 \mathrm{X}$ delay extension when we reduce $V_{d d}$ from $0.45 \mathrm{~V}$ to $0.3 \mathrm{~V}$ for FinFET circuits. In contrast, reducing $V_{d d}$ from $0.9 \mathrm{~V}$ to $0.7 \mathrm{~V}$ results in $2.3 \mathrm{X}$ energy reduction at the cost of $3 \mathrm{X}$ delay extension for $16 \mathrm{~nm}$ CMOS circuits. Therefore, one can observe a significant drop of energy-delay-products in Table 3 when reducing $V_{d d}$ of FinFET circuits.

Table 4 summarizes the fast operating frequencies we achieve for different sequential circuits and the corresponding power consumptions. One can observe from Table 4 that the clock frequency is improved by up to $5 \mathrm{X}$ and $25 \mathrm{X}$ by using the 5nm FinFET technology and applying the super-threshold supply voltage, against the $16 \mathrm{~nm}$ and $45 \mathrm{~nm}$ CMOS technology, respectively. Meanwhile, operating the circuits in the near-threshold supply voltage regime can reduce the average power consumption by up to $32 \mathrm{X}$ and $520 \mathrm{X}$, comparing to that of the $16 \mathrm{~nm}$ and $45 \mathrm{~nm}$ CMOS technology, respectively. Table 5 compares energy consumptions per clock of sequential benchmark circuits. Significant amount of energy reduction, up to $98 \mathrm{X}$ and $3000 \mathrm{X}$, are achieved by operating $5 \mathrm{~nm}$ FinFET circuits in the near-threshold regime against the $16 \mathrm{~nm}$ and $45 \mathrm{~nm}$ CMOS technology, respectively. While in the super-threshold regime, the corresponding energy reductions are up to $55 \mathrm{X}$ and $900 \mathrm{X}$, respectively.

We show the energy and delay of the 16-bit adder at different technology nodes in Figure 5, and energy per clock cycle and period of LEON2 SPARC processor in Figure 6. One can observe that $5 \mathrm{~nm}$ FinFET technology achieves much better circuit speed and lower energy consumption simultaneously, against the $16 \mathrm{~nm}$ and $45 \mathrm{~nm}$ CMOS technology. The near-threshold and super-threshold supply voltages provide a design trade-off between the circuit speed and the energy consumption.

\section{CONCLUSION}

FinFET became a promising VLSI technology for recent future due to its extraordinary properties. We adopted the most advanced 5nm FinFET device model and presented a design flow to build a standard cell library with multiple supply voltage regimes, considering both high performance and low power usages. The $5 \mathrm{~nm}$ FinFET standard cell library enabled static timing analysis, circuit synthesis, and dynamic voltage and frequency scaling at this technology node. The circuit synthesis results predicted that the $5 \mathrm{~nm}$ FinFET technology can achieve up to $40 \mathrm{X}$ circuit speed improvement as well as up to three orders of magnitude energy reduction against the $45 \mathrm{~nm}$ CMOS technology. We also observed a significant energy-delay-product drop by reducing the supply voltage of the $5 \mathrm{~nm}$ FinFET circuits.

\section{REFERENCES}

[1] R. Dreslinski, M. Wiekowski, D. Blaauw, D. Sylvester, and T. Mudge, "Near-threshold computing: reclaiming Moore's law through energy efficient integrated circuits," Proc. of IEEE, 2010.

[2] D. Markovic, C. Wang, L. Alarcon, T. Liu, and J. Rabaey, “Ultralowpower design in near-threshold region," Proc. of IEEE, 2010.

[3] A. Wang and A. Chandrakasan, "A $180 \mathrm{mV}$ FFT processor using circuit techniques," ISSCC, 2004.

[4] B. Zhai, D. Blaauw, D. Sylvester, and K. Flautner, "Theoretical and practical limits of dynamic voltage scaling," $D A C, 2004$.

[5] B. H. Calhoun, A. Wang, and A. Chandrakasan, "Modeling and sizing for minimum energy operation in subthreshold circuits," IEEE J. SolidState Circuits, 2005.

[6] X. Lin, Y. Wang, and M. Pedram, "Joint Sizing and Adaptive Independent Gate Control for FinFET Circuits Operating in Multiple Voltage Regimes Using the Logical Effort Method", ICCAD, 2013.

[7] T.-J. King, "FinFETs for nanoscale CMOS digital integrated circuits," ICCAD 2005, pp. 207-210.

[8] N. K. Jha, and D. Chen, Nanoelectronic Circuit Design, Springer Press, 2011.

[9] N. Lindert et. al., "Sub-60-nm quasi-planar FinFETs fabricated using a simplified process," IEEE on Electron Device Letters, vol.22, no.10, pp.487-489, Oct. 2001.

[10] E. J. Nowak et. al., "Turning silicon on its edge," IEEE Circuits and Devices Magazine, vol.20, no.1, pp.20-31, 2004.

[11] C. Wann, K. Noda, T. Tanaka, M. Yoshida, and C. Hu, "A comparative study of advanced MOSFET concepts," IEEE on Electron Devices, vol.43, no.10, pp.1742-1753, Oct 1996.

[12] L. Chang et. al., "Reduction of direct-tunneling gate leakage current in double-gate and ultra-thin body MOSFETs," IEDM, 2001.

[13] B. Yu et. al., "FinFET scaling to $10 \mathrm{~nm}$ gate length", IEDM 2002.

[14] A.R. Brown, A. Asenov, J. R. Watling, "Intrinsic fluctuations in sub 10$\mathrm{nm}$ double-gate MOSFETs introduced by discreteness of charge and matter," IEEE Transactions on Nanotechnology, vol.1, no.4, pp.195200, Dec 2002.

[15] S. Sinha, G. Yeric, V. Chandra, B. Cline, and Y. Cao, "Exploring Sub20nm FinFET Design with Predictive Technology Models", DAC, 2012.

[16] M. Dunga et al., BSIM-CMG: A Compact Model for Multi-Gate Transistors. Springer US, 2008.

[17] S. K. Gupta, W. Cho, A. A. Goud, K. Yogendra, and K. Roy, "Design space exploration of FinFETs in sub-10nm technologies for energyefficient near-threshold circuits," Device Research Conference (DRC), 2013.

[18] Synopsys Inc., Liberty Library Modeling, http://www.synopsys.com/ community/interoperability/pages/libertylibmodel.aspx.

[19] Synopsys Inc., HSPICE User Guide: Simulation and Analysis.

[20] Silvaco International., Introduction to Cell Characterization, [online] http://www.silvaco.com/content/kbase/cell_char_intro_090508.pdf.

[21] LEON2 SPRAC processor, [online] http://vlsicad.eecs.umich.edu/BK/ Slots/cache/www.gaisler.com/products/leon2/leon.html.

[22] 16nm PTM CMOS model, [online] http://ptm.asu.edu/latest.html.

[23] 45nm Open Cell Library, [online] http://www.nangate.com/?page_id=22

[24] FreePDK45, [online] http://www.eda.ncsu.edu/wiki/FreePDK45:Contents

[25] Design Compiler, Synopsys, [online] http://www.synopsys.com/Tools/ Implementation/RTLSynthesis/DCGraphical/Pages/default.aspx. 